ANNALES

POLONICI MATHEMATICI

$93.2(2008)$

\title{
Lifting adapted connections from foliated manifolds to higher order adapted frame bundles
}

\author{
by J. KuREK (Lublin) and W. M. Mikulski (Kraków)
}

\begin{abstract}
Let $(M, \mathcal{F})$ be a foliated manifold. We describe all natural operators $\mathcal{A}$ lifting $\mathcal{F}$-adapted (i.e. projectable in adapted coordinates) classical linear connections $\nabla$ on $(M, \mathcal{F})$ into classical linear connections $\mathcal{A}(\nabla)$ on the $r$ th order adapted frame bundle $P^{r}(M, \mathcal{F})$.
\end{abstract}

Introduction. All manifolds and maps are assumed to be of class $\mathcal{C}^{\infty}$.

A classical linear connection on a manifold $N$ is a $\mathbb{R}$-bilinear map $\nabla$ : $\mathcal{X}(N) \times \mathcal{X}(N) \rightarrow \mathcal{X}(N)$, where $\mathcal{X}(N)$ is the vector space of all vector fields on $N$, such that (1) $\nabla_{f X} Y=f \nabla_{X} Y$ and (2) $\nabla_{X} f Y=X f Y+f \nabla_{X} Y$ for any vector fields $X, Y \in \mathcal{X}(N)$ and all maps $f: N \rightarrow \mathbb{R}$. It is wellknown that classical linear connections $\nabla$ on $N$ are in canonical bijection with sections $\nabla: N \rightarrow Q N$ of the so-called connection bundle $Q(N)=$ $\left(\operatorname{id}_{T^{*} N} \otimes \pi_{1}\right)^{-1}\left(\operatorname{id}_{T N}\right) \subset T^{*} N \otimes J^{1} T N$ over $N$, where $\pi_{1}: J^{1} T N \rightarrow T N$ is the jet projection from the first jet prolongation $J^{1} T N$ of the tangent bundle $T N$. Every local diffeomorphism $f: N_{1} \rightarrow N_{2}$ induces canonically a fibred local diffeomorphism $Q f: Q\left(N_{1}\right) \rightarrow Q\left(N_{2}\right)$ covering $f$ (the restriction of $\left.T^{*} f \otimes J^{1} T f\right)$.

Let $\mathcal{F}_{o} l_{m, n}$ be the category of all $m+n$-dimensional foliated manifolds $(M, \mathcal{F})$ with $n$-dimensional foliations and their foliation respecting local diffeomorphisms. Given a $\mathcal{F}_{o l} l_{m, n}$ object $(M, \mathcal{F})$ we have the $r$ th order adapted frame bundle

$$
P^{r}(M, \mathcal{F})=\left\{j_{0}^{r} \varphi \mid \varphi:\left(\mathbb{R}^{m+n}, \mathcal{F}^{m, n}\right) \rightarrow(M, \mathcal{F}) \text { is a } \mathcal{F}_{o} l_{m, n} \text {-map }\right\}
$$

over $M$ of $(M \mathcal{F})$, where $\mathcal{F}^{m, n}=\left\{\{a\} \times \mathbb{R}^{n}\right\}_{a \in \mathbb{R}^{m}}$ is the standard $n$-dimensional foliation on $\mathbb{R}^{m+n}$. We see that $P^{r}(M, \mathcal{F})$ is a principal fibre bundle with the standard group $G_{m, n}^{r}=P^{r}\left(\mathbb{R}^{m, n}, \mathcal{F}^{m, n}\right)_{0}$ (with multiplication being composition of jets) acting on the right on $P^{r}(M, \mathcal{F})$ by the

2000 Mathematics Subject Classification: Primary 58A20.

Key words and phrases: foliated manifold, $(\mathcal{F}$-adapted $)$ classical linear connection, higher order adapted frame bundle, natural operator. 
composition of jets. Every $\mathcal{F}_{o} l_{m, n}$-map $\psi:\left(M_{1}, \mathcal{F}_{1}\right) \rightarrow\left(M_{2}, \mathcal{F}_{2}\right)$ induces a local fibred diffeomorphism (even a principal bundle local isomorphism) $P^{r} \psi: P^{r}\left(M_{1}, \mathcal{F}_{1}\right) \rightarrow P^{r}\left(M_{2}, \mathcal{F}_{2}\right)$ over $\psi$ given by $P^{r} \psi\left(j_{0}^{r} \varphi\right)=j_{0}^{r}(\psi \circ \varphi)$, $j_{0}^{r} \varphi \in P^{r}\left(M_{1}, \mathcal{F}_{1}\right)$.

Let $(M, \mathcal{F})$ be a $\mathcal{F}_{o l} l_{m, n}$-object. A vector field $X$ on $M$ is called an $i n$ finitesimal automorphism of $(M, \mathcal{F})$ if the flow $\{\operatorname{Expt} X\}$ of $X$ is formed by (locally defined) $\mathcal{F}_{0} l_{m, n^{-m a p s}}(M, \mathcal{F}) \rightarrow(M, \mathcal{F})$. Equivalently, a vector field on $M$ is an infinitesimal automorphism of $(M, \mathcal{F})$ iff $[X, Y]$ is tangent to $\mathcal{F}$ for any vector field $Y$ on $M$ tangent to $\mathcal{F}$. We denote by $\mathcal{X}(M, \mathcal{F})$ the Lie algebra of all infinitesimal automorphisms of $(M, \mathcal{F})$.

A classical linear connection $\nabla$ on $M$ is called $\mathcal{F}$-adapted if $\nabla_{X} Y \in$ $\mathcal{X}(M, \mathcal{F})$ for any $X, Y \in \mathcal{X}(M, \mathcal{F})$ and $\nabla_{U} W$ is tangent to $\mathcal{F}$ for any $U, W \in$ $\mathcal{X}(M, \mathcal{F})$ with $U$ or $W$ tangent to $\mathcal{F}$. (We observe in the proof of Lemma 1 that $\nabla$ is $\mathcal{F}$-adapted iff it is projectable in adapted coordinates.)

In the present paper we study how an $\mathcal{F}$-adapted classical linear connection $\nabla$ on a $\mathcal{F}_{o l} l_{m, n}$-object $(M, \mathcal{F})$ can induce a classical linear connection $\mathcal{A}(\nabla)$ on the $r$ th order adapted frame bundle $P^{r}(M, \mathcal{F})$. This problem is reflected in the concept of $\mathcal{F}_{o l_{m, n}}$-natural operators $\mathcal{A}: Q_{\mathcal{F}_{0} l_{m, n}} \rightsquigarrow Q P^{r}$ in the sense of [1]. We recall that a $\mathcal{F}_{O l_{m, n}}$ natural operator $\mathcal{A}: Q_{\mathcal{F}_{o l}, n} \rightsquigarrow Q P^{r}$ is a family of $\mathcal{F}_{o l} l_{m, n}$-invariant regular operators (functions)

$$
\mathcal{A}=\mathcal{A}_{(M, \mathcal{F})}: Q_{\mathcal{F}_{o l}}(M, \mathcal{F}) \rightarrow Q\left(P^{r}(M, \mathcal{F})\right)
$$

for any $\mathcal{F}_{\text {ol }}$ m,n-object $(M, \mathcal{F})$, where $Q_{\mathcal{F}_{o l}}(M, \mathcal{F})$ is the set of all $\mathcal{F}$-adapted classical linear connections on $(M, \mathcal{F})$ and $Q\left(P^{r}(M, \mathcal{F})\right)$ is the set of all classical linear connections on $P^{r}(M, \mathcal{F})$. The invariance means that if $\nabla_{1} \in$ $Q_{\mathcal{F} l}\left(M_{1}, \mathcal{F}_{1}\right)$ and $\nabla_{2} \in Q_{\mathcal{F}_{o l}}\left(M_{2}, \mathcal{F}_{2}\right)$ are $\varphi$-related for a $\mathcal{F}_{o l} l_{m, n}$-map $\varphi$ : $\left(M_{1}, \mathcal{F}_{1}\right) \rightarrow\left(M_{2}, \mathcal{F}_{2}\right)$, then $\mathcal{A}\left(\nabla_{1}\right)$ and $\mathcal{A}\left(\nabla_{2}\right)$ are $P^{r} \varphi$-related. The regularity means that $\mathcal{A}$ transforms smoothly parametrized families of $\mathcal{F}$-adapted classical linear connections into smoothly parametrized families of classical linear connections.

In Section 1 we give an example $\mathcal{A}^{0}: Q_{\mathcal{F}_{o l} l_{m, n}} \rightsquigarrow Q P^{r}$ of such a $\mathcal{F}_{o l_{m, n^{-}}}$ natural operator. Then we have

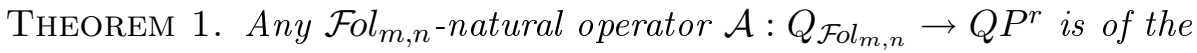
form

$$
\mathcal{A}(\nabla)=\mathcal{A}^{0}(\nabla)+\mathcal{C}(\nabla)
$$

for some unique $\mathcal{F}_{\text {ol }} l_{m, n}$-natural operator $\mathcal{C}: Q_{\mathcal{F}_{\text {ol }}, n} \rightarrow\left(T^{*} \otimes T^{*} \otimes T\right) P^{r}$ transforming $\mathcal{F}$-adapted classical linear connections $\nabla$ on $(M, \mathcal{F})$ into tensor fields $\mathcal{C}(\nabla)$ of type $(1,2)$ on $P^{r}(M, \mathcal{F})$.

In Sections 2 and 4 we describe explicitly all $\mathcal{F}_{o} l_{m, n}$-natural operators $\mathcal{C}: Q_{\mathcal{F}_{\text {ol }_{m, n}}} \rightsquigarrow\left(T^{*} \otimes T^{*} \otimes T\right) P^{r}$. (The definition of these operators is a direct modification of the one for $\mathcal{F}_{o} l_{m, n}$-natural operators $Q_{\mathcal{F}_{o l} l_{m, n}} \rightsquigarrow Q P^{r}$.) 
In the case $n=0$ we observe that $\mathcal{F}_{o} l_{m, 0}$ is (in an obvious way) equivalent to the category $\mathcal{M} f_{m}$ of $m$-dimensional manifolds and their local diffeomorphisms. Namely, we identify an $m$-manifold $M$ (an $\mathcal{M} f_{m^{-}}$object) with the foliated manifold $\left(M,\{\{a\}\}_{a \in M}\right)$ foliated by points (a $\mathcal{F}_{o l} l_{m, 0}$-object). Clearly, the bundle $P^{r}\left(M,\{\{a\}\}_{a \in M}\right)$ is (again in an obvious way) equivalent to the $r$ th order frame bundle $P^{r} M=\operatorname{inv} J_{0}^{r}\left(\mathbb{R}^{m}, M\right)$. Moreover, the

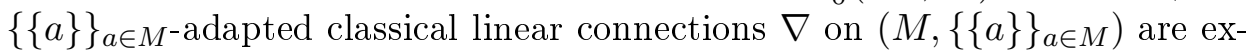
actly the classical linear connections $\nabla$ on $M$. Thus in the case $n=0$ the result of the present paper (almost) coincides with the result of the second author in [2]. In other words, the present paper is an (almost) extension of the result from [2] to foliated manifolds. We write "almost" because if $\nabla$ is a classical linear connection on $M$ then we have the complete lift $\nabla^{C}$ of $\nabla$ to $P^{r}(M)$ (we note that $P^{r}(M)$ is an open subset in $T_{m}^{r} M=J_{0}^{r}\left(\mathbb{R}^{m}, M\right.$ ) and we have the restriction of the complete lift $\nabla^{C}$ of $\nabla$ to the $m^{r}$-velocities bundle $T_{m}^{r} M$ of $M$ in the sense of Morimoto [3]). In [2], we use $\nabla^{C}$ instead of $\mathcal{A}^{0}(\nabla)$.

Up till now, for $n \geq 1$ no connections $\mathcal{A}(\nabla)$ on $P^{r}(M, \mathcal{F})$ coming from an $\mathcal{F}$-adapted one $\nabla$ on $(M, \mathcal{F})$ have been known. Thus the first main difficulty of the present paper is to construct a connection $\mathcal{A}^{0}(\nabla)$ on $P^{r}(M, \mathcal{F})$ from an $\mathcal{F}$-adapted classical linear connection $\nabla$ on $(M, \mathcal{F})$. In the construction of $\mathcal{A}^{0}(\nabla)$ we will use Lemma 1 , which we will also apply many times in next sections.

In the last section we present an alternative version of the main result.

\section{An example of a $\mathcal{F}_{o} l_{m, n}$-natural operator $\mathcal{A}^{0}: Q_{\mathcal{F}_{o l}, n} \rightsquigarrow Q P^{r}$.} To present such an example we need the following lemma.

Lemma 1. Let $\nabla$ be an $\mathcal{F}$-adapted classical linear connection on a $\mathcal{F}_{o l} l_{m, n^{-}}$ object $(M, \mathcal{F})$. Let $p=j_{0}^{r} \varphi \in\left(P^{r}(M, \mathcal{F})\right)_{x}, x \in M$. There exists a unique (germ of) $\nabla$-normal coordinate system $\psi^{\nabla, p}$ on $M$ with centre $x$ such that $\psi^{\nabla, p}:(M, \mathcal{F}) \rightarrow\left(\mathbb{R}^{m+n}, \mathcal{F}^{m, n}\right)$ is a locally defined $\mathcal{F}_{\text {ol }}$ m,n-map and we have $P^{1}\left(\psi^{\nabla, p}\right)\left(j_{0}^{1} \varphi\right)=j_{0}^{1}\left(\operatorname{id}_{\mathbb{R}^{m+n}}\right)$.

Proof. Clearly, for a vector field $X \in \mathcal{X}\left(\mathbb{R}^{m+n}\right)$ we have $X \in \mathcal{X}\left(\mathbb{R}^{m+n}\right.$, $\left.\mathcal{F}^{m+n}\right)$ iff the flow of $X$ is formed by local fibred isomorphisms of the trivial bundle $q: \mathbb{R}^{m} \times \mathbb{R}^{n} \rightarrow \mathbb{R}^{m}$, or (equivalently) iff $X$ is $q$-projectable (i.e. there exists a unique vector field $\underline{X}$ on $\mathbb{R}^{m} q$-related to $X$ ). Then a classical linear connection $\nabla$ on $\mathbb{R}^{m+n}$ is $\mathcal{F}^{m, n}$-adapted iff $\nabla_{X} Y$ is $q$-projectable for any $q$-projectable vector fields $X, Y$ on $\mathbb{R}^{m} \times \mathbb{R}^{n}$ and $\nabla_{U} W$ is vertical if $U, V$ are $q$-projectable and $U$ or $W$ is vertical, or (equivalently) iff $\nabla$ is $q$-projectable (i.e. there exists a unique classical linear connection $\underline{\nabla}$ on $\mathbb{R}^{m} q$-related to $\nabla$ ).

We can assume that $(M, \mathcal{F})=\left(\mathbb{R}^{m+n}, \mathcal{F}^{m, n}\right), x=0$ and $j_{0}^{1} \varphi=j_{0}^{1}\left(\operatorname{id}_{\mathbb{R}^{m+n}}\right)$. By the above considerations, the exponent $\operatorname{Exp}_{\nabla, 0}$ of $\nabla$ at $0 \in \mathbb{R}^{m+n}$ is 
$q$-related to $\operatorname{Exp}_{\nabla, 0}$ of $\underline{\nabla}$ at $0 \in \mathbb{R}^{m}$ (because $\nabla$-geodesics project via $q$ onto $\underline{\nabla}$-geodesics as $\bar{\nabla}$ and $\underline{\nabla}$ are $q$-related and $q$ is a surjective submersion). Then $\psi^{\nabla, p}=\operatorname{Exp}_{\nabla, 0}^{-1}$ is a unique $\nabla$-normal coordinate system on $\mathbb{R}^{m+n}$ with centre 0 such that $P^{1}\left(\psi^{\nabla, p}\right)\left(j_{0}^{1}(\mathrm{id})\right)=j_{0}^{1}(\mathrm{id})$ and $\psi^{\nabla, p}:\left(\mathbb{R}^{m+n}, \mathcal{F}^{m, n}\right) \rightarrow$ $\left(\mathbb{R}^{m+n}, \mathcal{F}^{m, n}\right)$ is a locally defined $\mathcal{F}_{o} l_{m, n^{-m a p}}$

We are in a position to present an example of a $\mathcal{F}_{o} l_{m, n}$-natural operator $\mathcal{A}^{0}: Q_{\mathcal{F}_{\text {ol }}, n} \rightsquigarrow Q P^{r}$. We fix an arbitrary classical linear connection $\lambda^{0}$ on $P^{r}\left(\mathbb{R}^{m+n}, \mathcal{F}^{m, n}\right)$ (a section of the connection bundle $Q\left(P^{r}\left(\mathbb{R}^{m+n}, \mathcal{F}^{m, n}\right)\right)$ $\left.\rightarrow P^{r}\left(\mathbb{R}^{m+n}, \mathcal{F}^{m, n}\right)\right)$.

ExAmple 1. Let $\nabla \in Q(M, \mathcal{F})$ be an $\mathcal{F}$-adapted classical linear connection on a $\mathcal{F}_{o l} l_{m, n}$-object $(M, \mathcal{F})$. We define a classical linear connection $\mathcal{A}^{0}(\nabla)$ on $P^{r}(M, \mathcal{F})$ (a section $\mathcal{A}^{0}(\nabla): P^{r}(M, \mathcal{F}) \rightarrow Q\left(P^{r}(M, \mathcal{F})\right)$ ) as follows. Let $p=j_{x}^{r} \varphi \in\left(P^{r}(M, \mathcal{F})\right)_{x}, x \in M$. Let $\psi^{\nabla, p}$ be the unique (germ of) $\nabla$-normal coordinate system on $M$ with centre $x$ such that $\psi^{\nabla, p}:(M, \mathcal{F}) \rightarrow$ $\left(\mathbb{R}^{m+n}, \mathcal{F}^{m, n}\right)$ is a locally defined $\mathcal{F}_{o} l_{m, n}$-map and $P^{1}\left(\psi^{\nabla, p}\right)\left(j_{0}^{1} \varphi\right)=j_{0}^{1}(\mathrm{id})$ (see Lemma 1). We define

$$
\mathcal{A}^{0}(\nabla)(p):=Q\left(P^{r}\left(\left(\psi^{\nabla, p}\right)^{-1}\right)\right)\left(\lambda^{0}\left(P^{r}\left(\psi^{\nabla, p}\right)(p)\right)\right) .
$$

This definition is correct because the germ of $\psi^{\nabla, p}$ at $x$ is uniquely determined and we can apply the functor $P^{r}$ to $\psi^{\nabla, p}$ because $\psi^{\nabla, p}$ is a $\mathcal{F}_{o} l_{m, n^{-m a p}}$. Therefore the family $\mathcal{A}^{0}: Q_{\mathcal{F}_{o l} l_{m, n}} \rightsquigarrow Q P^{r}$ is a $\mathcal{F}_{o} l_{m, n}$-natural operator.

2. The $\mathcal{F}_{o l} l_{m, n}$-natural operators $Q_{\mathcal{F}_{O l_{m, n}}} \rightsquigarrow T^{(0,0)} P^{r}$. Let

$$
\theta:=j_{0}^{1}\left(\operatorname{id}_{\mathbb{R}^{m+n}}\right) \in\left(P^{1}\left(\mathbb{R}^{m+n}, \mathcal{F}^{m, n}\right)\right)_{0} .
$$

Let $\left(P^{r}\left(\mathbb{R}^{m+n}, \mathcal{F}^{m, n}\right)\right)_{\theta}=\left\{j_{0}^{r} \varphi \in\left(P^{r}\left(\mathbb{R}^{m+n}, \mathcal{F}^{m, n}\right)\right)_{0} \mid j_{0}^{1} \varphi=\theta\right\}$. Let $S^{s}$ $(s \in \mathbb{N} \cup\{\infty\})$ be the vector space of all $s$-jets at $0 \in \mathbb{R}^{m+n}$ of $\mathcal{F}^{m, n}$-adapted classical linear connections $\nabla$ on $\left(\mathbb{R}^{m+n}, \mathcal{F}^{m, n}\right)$ (or equivalently, of all $s$-jets at $0 \in \mathbb{R}^{m+n}$ of projectable classical linear connections on the trivial bundle $\mathbb{R}^{m} \times \mathbb{R}^{n} \rightarrow \mathbb{R}^{m}$ ) given by the Christoffel symbols $\Gamma_{j k}^{i}: \mathbb{R}^{m+n} \rightarrow \mathbb{R}$ satisfying

$$
\sum_{j, k=1}^{m+n} \Gamma_{j k}^{i}(x) x^{j} x^{k}=0 \quad \text { for } i=1, \ldots, m+n .
$$

Equivalently, $S^{s}$ is the space of all $s$-jets at 0 of $\mathcal{F}^{m, n}$-adapted classical linear connections $\nabla$ on $\left(\mathbb{R}^{m+n}, \mathcal{F}^{m, n}\right)$ such that the usual coordinate system $x^{1}, \ldots, x^{m+n}$ on $\mathbb{R}^{m+n}$ is a normal coordinate system for $\nabla$ with centre 0 . (The equivalence is almost clear if we remember the well-known differential equations for geodesics and apply the fact that $\nabla$-geodesics passing through the centre of $\nabla$-normal coordinates are straight lines in these coordinates.)

Let us consider a function $\mu: S^{\infty} \times\left(P^{r}\left(\mathbb{R}^{m+n}, \mathcal{F}^{m, n}\right)\right)_{\theta} \rightarrow \mathbb{R}$ satisfying the following local finite determination property: 
(*) For any $\varrho \in S^{\infty}$ and $\sigma \in\left(P^{r}\left(\mathbb{R}^{m+n}, \mathcal{F}^{m, n}\right)\right)_{\theta}$, we can find an open neighbourhood $V \subset\left(P^{r}\left(\mathbb{R}^{m+n}, \mathcal{F}^{m, n}\right)\right)_{\theta}$ of $\sigma$, an open neighbourhood $U \subset S^{\infty}$ of $\varrho$, a natural number $s$ and a smooth map $f$ : $\pi_{s}(U) \times V \rightarrow \mathbb{R}$ such that $\mu=f \circ\left(\pi_{s} \times \mathrm{id}_{V}\right)$ on $U \times V$, where $\pi_{s}: J^{\infty} \rightarrow J^{s}$ is the jet projection.

An example of a $\mu$ with property $(*)$ is the pull-back of a map $\widetilde{\mu}: S^{s} \times$ $\left(P^{r}\left(\mathbb{R}^{m+n}, \mathcal{F}^{m, n}\right)\right)_{\theta} \rightarrow \mathbb{R}$ for finite $s$ with respect to the projection $\pi_{s} \times$ id : $S^{\infty} \times\left(P^{r}\left(\mathbb{R}^{m+n}, \mathcal{F}^{m, n}\right)\right)_{\theta} \rightarrow S^{s} \times\left(P^{r}\left(\mathbb{R}^{m, n}, \mathcal{F}^{m, n}\right)\right)_{\theta}$.

EXAMPLE 2. Given an $\mathcal{F}$-adapted classical linear connection $\nabla$ on a $\mathcal{F}_{\text {ol }} l_{m, n}$-object $(M, \mathcal{F})$, we define a smooth map $\mathcal{B}^{\langle\mu\rangle}(\nabla): P^{r}(M, \mathcal{F}) \rightarrow \mathbb{R}$ by

$$
\mathcal{B}^{\langle\mu\rangle}(\nabla)(p):=\mu\left(j_{0}^{\infty}\left(\psi_{*}^{\nabla, p} \nabla\right), P^{r}\left(\psi^{\nabla, p}\right)(p)\right),
$$

$p=j_{0}^{r} \varphi \in\left(P^{r}(M, \mathcal{F})\right)_{x}, x \in M$, where $\psi^{\nabla, p}:(M, \mathcal{F}) \rightarrow\left(\mathbb{R}^{m+n}, \mathcal{F}^{m, n}\right)$ is as in Lemma 1 for $\nabla$ and $p$. The definition is correct because $\operatorname{germ}_{x}\left(\psi^{\nabla, p}\right)$ is uniquely determined. The correspondence $\mathcal{B}^{\langle\mu\rangle}: Q_{\mathcal{F}_{\text {ol }} m, n} \rightsquigarrow T^{(0,0)} P^{r}$ is a $\mathcal{F}_{o l} l_{m, n}$-natural operator transforming $\mathcal{F}$-adapted classical linear connections on $\mathcal{F}_{o l} l_{m, n^{-}}$objects $(M, \mathcal{F})$ into maps $\mathcal{B}^{\langle\mu\rangle}(\nabla): P^{r}(M, \mathcal{F}) \rightarrow \mathbb{R}$. (The definition of $\mathcal{F}_{o} l_{m, n}$-natural operators $Q_{\mathcal{F}_{\text {ol }} m, n} \rightsquigarrow T^{(0,0)} P^{r}$ is a direct modification of the one of $\mathcal{F}_{m, n}$-natural operators $Q_{\mathcal{F}_{\text {ool }}, n} \rightsquigarrow Q P^{r}$.)

Proposition 1. Any $\mathcal{F}_{\text {ol }} l_{m, n}$-natural operator $\mathcal{B}: Q_{\mathcal{F}_{o l}, n} \rightsquigarrow T^{(0,0)} P^{r}$ is equal to $\mathcal{B}^{\langle\mu\rangle}$ for a unique map $\mu: S^{\infty} \times\left(P^{r}\left(\mathbb{R}^{m+n}, \mathcal{F}^{m, n}\right)\right)_{\theta} \rightarrow \mathbb{R}$ with the above-mentioned property.

Proof. Let $\mathcal{B}$ be an operator in question. Define a map $\mu: S^{\infty} \times\left(P^{r}\left(\mathbb{R}^{m+m}\right.\right.$, $\left.\left.\mathcal{F}^{m, n}\right)\right)_{\theta} \rightarrow \mathbb{R}$ by

$$
\mu\left(j_{0}^{\infty}(\nabla), \sigma\right)=\mathcal{B}(\nabla)_{\sigma} .
$$

Then by the non-linear Peetre theorem [1], $\mu$ has property $(*)$. Clearly, $\mathcal{B}=$ $\mathcal{B}^{\langle\mu\rangle}$.

3. The parallelism on $P^{r}(M, \mathcal{F})$ from an $\mathcal{F}$-adapted classical linear connection $\nabla$ on $(M, \mathcal{F})$. Let $\nabla$ be an $\mathcal{F}$-adapted classical linear connection $\nabla$ on $(M, \mathcal{F})$.

EXAMPLE 3. For $i=1, \ldots, m+n$, we define a vector field $A^{i}(\nabla)$ on $P^{r}(M, \mathcal{F})$ as follows. Let $p=j_{0}^{r} \varphi \in\left(P^{r}(M, \mathcal{F})\right)_{x}, x \in M$. Let $\psi^{\nabla, p}$ : $(M, \mathcal{F}) \rightarrow\left(\mathbb{R}^{m+n}, \mathcal{F}^{m, n}\right)$ be as in Lemma 1 for $\nabla$ and $p$. We put

$$
A^{i}(\nabla)(p)=T P^{r}\left(\left(\psi^{\nabla, p}\right)^{-1}\right)\left(\mathcal{P}^{r}\left(\frac{\partial}{\partial x^{i}}\right)\left(P^{r}\left(\psi^{\nabla, p}\right)(p)\right)\right),
$$

where $\partial / \partial x^{i}$ are the canonical vector fields on $\mathbb{R}^{m+n}$ (they are infinitesimal automorphisms of $\left.\left(\mathbb{R}^{m+n}, \mathcal{F}^{m, n}\right)\right)$ and where $\mathcal{P}^{r} X$ means the flow lifting of an infinitesimal automorphism $X \in \mathcal{X}\left(N, \mathcal{F}_{1}\right)$ to $P^{r}\left(N, \mathcal{F}_{1}\right)$. $\left(\mathcal{P}^{r} X\right.$ is given 
by the flow $\left\{P^{r}(\operatorname{Expt} X)\right\}$, where $\{\operatorname{Expt} X\}$ is the flow of $X$. We can apply the functor $P^{r}$ to $\operatorname{Expt} X$ because $\operatorname{Expt} X$ is a $\mathcal{F}_{o l} l_{m, n}$-map as $X$ is an infinitesimal automorphism.) The correspondence $A^{i}: Q_{\mathcal{F}_{\text {ol }}, n} \rightsquigarrow T P^{r}$ is a $\mathcal{F}_{o} l_{m, n}$-natural operator (transforming $\mathcal{F}$-adapted classical linear connections on $(M, \mathcal{F})$ into vector fields on $\left.P^{r}(M, \mathcal{F})\right)$.

EXAMPLE 4. Let $G_{m, n}^{r}=P^{r}\left(\mathbb{R}^{m+n}, \mathcal{F}^{m, n}\right)_{0}$ be the Lie group as in the Introduction. Let $\left\{E_{\alpha}\right\}$ be a basis in the Lie algebra $\mathcal{L}\left(G_{m, n}^{r}\right)$. Let $\left(E_{\alpha}\right)^{*}$ be the fundamental vector field corresponding to $E_{\alpha}$ on the principal $G_{m, n^{-}}^{r}$ bundle $P^{r}(M, \mathcal{F})$. The correspondence $\left(E_{\alpha}\right)^{*}: Q_{\mathcal{F}_{o l} l_{m, n}} \rightsquigarrow T P^{r}$ is a $\mathcal{F}_{o l} l_{m, n^{-}}$ natural operator (independent of $\nabla$ ).

Proposition 2. Given an $\mathcal{F}$-adapted classical linear connection $\nabla$ on $(M, \mathcal{F})$, the vector fields $A^{i}(\nabla)$ and $\left(E_{\alpha}\right)^{*}$ for $i=1, \ldots, m+n$ and $\alpha=$ $1, \ldots, \operatorname{dim}\left(G_{m, n}^{r}\right)$ form a basis over $\mathcal{C}^{\infty}\left(P^{r}(M, \mathcal{F})\right)$ of the module of all vector fields on $P^{r}(M, \mathcal{F})$.

Proof. This is a simple observation.

4. The $\mathcal{F}_{o l} l_{m, n}$-natural operators $Q_{\mathcal{F}_{o l} l_{m, n}} \rightsquigarrow\left(T^{*} \otimes T^{*} \otimes T\right) P^{r}$. The space of all $\mathcal{F}_{o l_{m, n}}$-natural operators $Q_{\mathcal{F}_{o l} l_{m, n}} \rightsquigarrow\left(T^{*} \otimes T^{*} \otimes T\right) P^{r}$ transforming $\mathcal{F}$-adapted classical linear connections on $(M, \mathcal{F})$ into tensor fields of type $(1,2)$ on $P^{r}(M, \mathcal{F})$ is (in an obvious way) a module over the algebra of all $\mathcal{F}_{\text {ol }} l_{m, n}$-natural operators $Q_{\mathcal{F} o l_{m, n}} \rightsquigarrow T^{(0,0)} P^{r}$ (classified in Section 2).

Proposition 3. The module of all $\mathcal{F}_{\text {ol }} m_{m, n}$-natural operators $Q_{\mathcal{F}_{\text {ol }} m, n}$ $\rightsquigarrow\left(T^{*} \otimes T^{*} \otimes T\right) P^{r}$ is free and $\left(m+n+\operatorname{dim}\left(G_{m, n}^{r}\right)\right)^{3}$-dimensional. All (suitable) tensor products of $A^{i},\left(E_{\alpha}\right)^{*},\left(A^{i}\right)^{D}$ and $\left(\left(E_{\alpha}\right)^{*}\right)^{D}$ form a basis in this module, where given an $\mathcal{F}$-adapted classical linear connection $\nabla$ on $(M, \mathcal{F})$, $\left(A^{i}(\nabla),\left(E_{\alpha}\right)^{*}\right)$ is a basis of vector fields on $P^{r}(M, \mathcal{F})$ as in Section 3 and $\left(A^{i}(\nabla)^{D},\left(\left(E_{\alpha}\right)^{*}\right)^{D}\right)$ is the dual basis of 1 -forms on $P^{r}(M, \mathcal{F})$.

Proof. Let $\mathcal{C}: Q_{\mathcal{F}_{\text {ol }} m, n} \rightsquigarrow\left(T^{*} \otimes T^{*} \otimes T\right) P^{r}$ be a $\mathcal{F}_{o l} l_{m, n}$-natural operator. For any $\mathcal{F}$-adapted classical linear connection $\nabla$ on $(M, \mathcal{F})$ we can write

$$
\mathcal{C}(\nabla)=\sum_{k} \lambda_{k}(\nabla) F^{k}(\nabla)
$$

where $\left(F^{k}(\nabla)\right)$ is the obvious basis of $(1,2)$-tensor fields on $P^{r}(M, \mathcal{F})$ induced by the basis $\left(A^{i}(\nabla),\left(E_{\alpha}\right)^{*}\right)$, and the maps $\lambda_{k}(\nabla): P^{r}(M, \mathcal{F}) \rightarrow \mathbb{R}$ are uniquely determined. Because of the invariance of $\mathcal{C}$ with respect to $\mathcal{F}_{0} l_{m, n}$-maps, $\lambda_{k}: Q_{\mathcal{F}_{0} l_{m, n}} \rightsquigarrow T^{(0,0)} P^{r}$ are $\mathcal{F}_{o} l_{m, n}$-natural operators.

5. Another version of the main theorem. We end this note by the following alternative description of all $\mathcal{F}_{o l_{m, n}}$-natural operators $\mathcal{A}$ : $Q_{\mathcal{F}_{o} l_{m, n}} \rightsquigarrow Q P^{r}$. 
We use the notation of Section 2. Let $\nu: S^{\infty} \times\left(P^{r}\left(\mathbb{R}^{m+n}, \mathcal{F}^{m, n}\right)\right)_{\theta} \rightarrow$ $Q\left(P\left(\mathbb{R}^{m+n}, \mathcal{F}^{m, n}\right)\right)$ be a map such that a local finite determination property quite similar to $(*)$ for $\mu$ from Section 2 is satisfied. Additionally we assume that $\pi \circ \nu(\sigma, p)=p$ for any $(\sigma, p)$, where $\pi$ is the projection of the connection bundle.

EXAmple 5. Let $\nabla$ be an $\mathcal{F}$-adapted classical linear connection on a

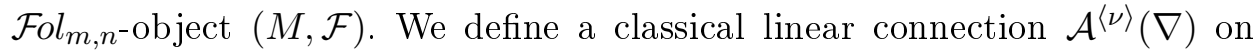
$P^{r}(M, \mathcal{F})$ as follows. Let $p=j_{0}^{r} \varphi \in\left(P^{r}(M, \mathcal{F})\right)_{x}, x \in M$. Let $\psi^{\nabla, p}$ : $(M, \mathcal{F}) \rightarrow\left(\mathbb{R}^{m+n}, \mathcal{F}^{m, n}\right)$ be as in Lemma 1 for $\nabla$ and $p$. We put

$$
\mathcal{A}^{\langle\nu\rangle}(\nabla)(p)=Q\left(P^{r}\left(\left(\psi^{\nabla, p}\right)^{-1}\right)\right)\left(\nu\left(j_{0}^{\infty}\left(\psi_{*}^{\nabla, p} \nabla\right), P^{r}\left(\psi^{\nabla, p}\right)(p)\right)\right) .
$$

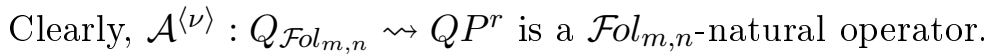

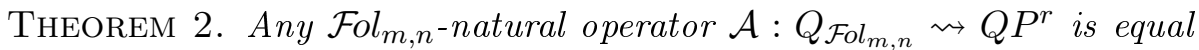
to $\mathcal{A}^{\langle\nu\rangle}$ for some $\nu$ as in Example 5.

Proof. The proof is quite similar to the one of Proposition 1.

\section{References}

[1] I. Kolář, P. W. Michor and J. Slovák, Natural Operations in Differential Geometry, Springer, 1993.

[2] W. M. Mikulski, Natural liftings of connections to the rth order frame bundle, Demonstratio Math. 40 (2007), 481-484.

[3] A. Morimoto, Liftings of some types of tensor fields and connections to tangent bundles of $p^{r}$-velocities, Nagoya Math. J. 40 (1970), 13-31.

Institute of Mathematics

Maria Curie-Skłodowska University

Pl. M. Curie-Skłodowskiej 1

20-031 Lublin, Poland

E-mail: kurek@hektor.umcs.lublin.pl
Institute of Mathematics Jagiellonian University Reymonta 4

30-059 Kraków, Poland E-mail: mikulski@im.uj.edu.pl

Received 24.7.2007

and in final form 5.10.2007 